\title{
Handoff Modeling in Cellular CDMA with Finite Sources and State-Dependent Bandwidth Requirements
}

\author{
Vassilios G. Vassilakis*, Ioannis D. Moscholios ${ }^{\dagger}$, John S. Vardakas ${ }^{\ddagger}$, Michael D. Logothetis ${ }^{\S}$ \\ * Dept. of Electronic Engineering, University of Surrey, Guildford, U.K. \\ $\dagger$ Dept. of Informatics \& Telecommunications, University of Peloponnese, Tripolis, Greece \\ $\ddagger$ Iquadrat, Barcelona, Spain \\ $\S$ Dept. of Electrical \& Computer Engineering, University of Patras, Patras, Greece
}

\begin{abstract}
It is envisioned that small cells are going to play an important role in future cellular networks. Due to limited coverage areas of small cells and increased user mobility, a proper analysis and evaluation of handoff traffic is of major importance. In this paper, we propose a novel teletraffic model for the analysis of handoff traffic in cellular CDMA networks. When the cell load is above a predefined threshold, we allow the handoff calls to reduce their bandwidth requirements in order to avoid blocking. Our model also realistically assumes a finite number of traffic sources in the cell and enables analytical determination of uplink blocking probabilities for new and handoff calls. To this end, the CDMA system has been described as a continuous-time Markov chain in order to derive an efficient recursive formula for the calculation of system state probabilities. The proposed analytical model has been validated through simulation experiments, which show good accuracy of the derived results.
\end{abstract}

\section{INTRODUCTION}

The family of Code-Division Multiple Access (CDMA) techniques has been successfully used as radio link interface in the current generation mobile networks. Furthermore, CDMA techniques are expected to play an important role in future fifth generation (5G) networks [1], [2]. According to the CDMA radio communication, signals of different Mobile Users (MUs) are distinguished by different codes. The codes used in practice are non-orthogonal and, as a consequence, a new MU arriving to a cell will cause interference to other MUs in the same and neighbouring cells. Hence, in order to protect the Quality-ofService (QoS) of existing MUs, Call Admission Control (CAC) is performed at the Base Station (BS) upon a call arrival. This may cause blocking of a newly arriving call.

In this paper, we consider two types of call blocking: newcall blocking and handoff-call blocking. The first type refers to the call blocking upon the initial connection establishment. The second type refers to the blocking of in-service calls when they move from one cell to another. Handoff is the process of moving between neighbouring cells, while a call is in progress. In that case, the BS of the target cell takes the control from the BS of the source cell. The CAC policy, used at the BSs, is expected to guarantee that the handoff-call blocking probability will be significantly lower than the new-call blocking.

A number of works study cellular CDMA systems and derive new-call blocking probabilities. In [3], the calculation of call blocking probabilities in the uplink of W-CDMA is studied. The call arrival process is assumed to be Poisson and calls have fixed bandwidth requirements. This work was extended in [4], by modeling elastic and adaptive services, and in [5], [6] by considering a finite number of traffic sources. In [7], the authors propose and evaluate an efficient CAC policy for CDMA networks. The system has been modeled as a CTMC and a recurrent formula for calculation of system state probabilities has been derived. The model of [5] has been extended in [8] to incorporate elastic services and in [9], [10] to enable interference cancellation. In [11], the blocking performance in the downlink of CDMA is analysed, based on Markov chain modelling. Some of the aforementioned models have been extended for the analysis of handoff-call blocking probabilities. In [12] and [13], teletraffic models for W-CDMA networks with a soft handoff mechanism have been proposed. In [14], the authors extend the model of [5] by incorporating the calculation of handoff-call blocking probabilities. In [15], the model of [3] has been enhanced with a CAC for handoff traffic. In [16], a teletraffic model for CDMA supporting batched Poisson call arrivals is proposed. In [17], an extensive survey of handoff decision algorithms is presented.

In this paper, we model and analyse the uplink of cellular CDMA systems including handoff traffic. We describe call arrivals/departures as a Continuous-Time Markov Chain (CTMC). Next, we propose an efficient and recurrent formula for the calculation of system state probabilities, and consequently, call blocking probabilities. In particular, we extend the model of [15] by enabling two contingency statedependent bandwidth requirements for arriving handoff calls. If the system is heavily loaded (above a predefined threshold), then an arriving call will request less bandwidth compared with the case of lightly loaded systems. This is based on the single-threshold model [18] that has been proposed for wired connection-oriented networks. We extend this model by considering handoff traffic, state-dependent call bandwidth requirements, soft network capacity, and other peculiarities of CDMA cellular systems. Also, the infinite number of traffic sources assumed in [15], is considered unrealistic, especially in the environment of small cells. Therefore, we incorporate a finite number of traffic sources in our proposed model.

This paper is structured as follows. In Section II, we describe our proposed model for cellular CDMA systems. In Section III, we analyse the model using a CTMC and derive equations for efficient calculation of new-call and handoff- 
call uplink blocking probabilities. In Section IV, we evaluate the accuracy of our proposed model by means of computer simulations. Finally, we conclude in Section V.

\section{SYSTEM DESCRIPTION}

Consider a CDMA system that supports $K$ independent service-classes. We consider two different types of calls: new and handoff. The first type refers to calls that originate inside the reference cell. The second type includes calls that originate in a neighbouring cell and are transferred to the reference cell through the handoff process.

Each service-class $k(k=1, \ldots, K)$ has a finite number of new traffic sources, denoted by $S_{k, N}$, and handoff traffic sources, denoted by $S_{k, H}$. A traffic source may be either busy, having a call in progress, or idle, waiting for the user to start a call. The offered traffic-load per idle source of service-class $k$ new calls is denoted by $a_{k, N}$. In a similar way, the offered traffic-load per idle source of service-class $k$ handoff calls is denoted by $a_{k, H}$. In order to avoid repetitions, in some cases we will collectively refer to the offered traffic-load of both new and handoff calls as $a_{k, t}$, where $t$ stands for type and $t \in$ $\{N, H\}$. Also, throughout the paper, we will use the indices $N$ and $H$ to refer to new and handoff calls, respectively.

An in-service call alternates between transmitting (active) and silent (passive) periods. To model this behaviour, we define the activity factor, $v_{k}$, of a service-class $k$ call as the percentage of the duration of active periods over the total call duration. We also define the Block Error Rate (BER) parameter, $\left(E_{b} / N_{0}\right)_{k}$, of service-class $k$ as the signal energy per bit divided by the noise spectral density, required to meet a predefined BER. We also make the reasonable assumption that the activity factor and the BER parameter are the same for both new and handoff calls of a given service-class.

As it is known, the capacity of CDMA-based systems is limited by the multiple access interference. To model this characteristic, the cell load, $n$, which represents the total occupied cell radio resource, has been introduced [3]. The cell load consists of the intra-cell load, $n_{\text {intra }}$, (caused my MUs of the reference cell) and the inter-cell load, $n_{\text {inter }}$, (caused my MUs of neighbouring cells).

For the service-class $k$ new calls we assume that the transmission bit rate, $R_{k, N}$, is fixed throughout the call duration. However, the transmission bit rate of service-class $k$ handoff calls may take two different values, depending on the cell load, $n$, at the moment of call arrival. In particular, if the cell load is below a predefined threshold, $n_{k, H}$, then the call requests its peak transmission rate, $R_{k, H P}$. On the other hand, if $n \geq n_{k, H}$, then the call requests a lower transmission rate, $R_{k, H L}$. Throughout the paper, we will use the indices $P$ and $L$ to refer to peak and low transmission rates, respectively, for handoff calls. Also, we will use the variable $r$ (stands for rate) to refer collectively to both peak and low transmission rates; that is, $r \in\{P, L\}$.

When a call (new or handoff) is accepted in the cell, it increases the cell load by the so-called call load factor, $L_{k, t r}$, which is defined as follows and similarly to [15]:

$$
L_{k, t r}=\frac{\left(E_{b} / N_{0}\right)_{k} R_{k, t r}}{W+\left(E_{b} / N_{0}\right)_{k} R_{k, t r}}
$$

where, as usual, $t \in\{N, H\}, r \in\{P, L\}$, and $W$ is the chip rate. A typical value in W-CDMA systems is $W=3.84 \mathrm{Mcps}$.

We consider the following CAC policy for the acceptance of a service-class $k$, type $t$, rate $r$ call:

$$
n_{\text {intra }}+n_{\text {inter }}+L_{k, t r} \leq n_{\max , t}
$$

That is, the call is accepted if and only if, after the acceptance, the cell load is not going to exceed the threshold $n_{\max , t}$.

Note that the rate threshold $n_{k, H}$ is used to select the requested transmission rate of handoff calls, whereas the $C A C$ threshold $n_{\max , t}$ is used to decide whether to block/accept a new or a handoff call.

\section{Calculating Blocking Probabilities}

\section{A. Local Blocking Probabilities}

Due to the CAC of (2), some of the arriving calls in the cell may be blocked by the BS. The probability that a call of service-class $k$, type $t$, and rate $r$ is blocked when arriving at an instant with intra-cell load, $n_{\text {intra }}$, is called Local Blocking Probability (LBP) and defined as:

$$
\beta_{k, t r}\left(n_{\text {intra }}\right)=\operatorname{Pr}\left[n_{\text {intra }}+n_{\text {inter }}+L_{k, t r}>n_{\max , t}\right]
$$

Following similar calculations as in [15], we get:

$$
\beta_{k, t r}\left(n_{\text {intra }}\right)=1-C D F_{n_{\text {inter }}}(x), \text { for } x \geq 0
$$

where $x=n_{\max , t}-n_{\text {intra }}-L_{k, t r}$ and $C D F_{n_{\text {inter }}}()$ is the cumulative distribution function of $n_{\text {inter }}$.

\section{B. State Transition Diagram}

In this subsection, we describe the call arrival and departure process as a CTMC and derive the corresponding state transition diagram. The first step is to discretize the cell load, the intra-cell load, the call load factor, and the rate threshold with the aid of the basic discretization unit, $g$, as follows:

$$
j=\left\lfloor\frac{n}{g}\right\rfloor, c=\left\lfloor\frac{n_{\text {intra }}}{g}\right\rfloor, b_{k, t r}=\left\lfloor\frac{L_{k, t r}}{g}\right\rfloor, J_{k, H}=\left\lfloor\frac{n_{k, H}}{g}\right\rfloor
$$

where the floor function $\lfloor x\rfloor$ gives the largest integer less than or equal to $x$.

The physical meaning of the aforementioned parameters can be explained with the aid of a simple example. Assume that a cell has three MUs and all of them are in the middle of a voice conversation, which requires 1 resource unit (RU) (equivalent e.g. to a $64 \mathrm{Kbps}$ data rate). The number of required RUs by a call, corresponds to the parameter $b_{k, t r}$. At a given moment, some MUs may be active (i.e. transmitting), whereas other will be passive (i.e. receiving or idle). The parameter $j$, considered as the system state, represents the number of RUs occupied in the cell, assuming that all MUs are active. Therefore, in this example $j=3 b_{k, t r}=3$. The parameter $c$, defined as the resource occupancy, represents the actual number of occupied RUs (i.e., takes into account only active users). In this example, $c=0$ if all MUs are passive, $c=1$ if one MU is active and two MUs are passive, etc. It is clear from the above that $j$ depends on the number of MUs and the services they are using, whereas $c$ also depends on the user activity. Also note that $0 \leq c \leq j$. When all MUs are passive, 
we have $c=0$; when all MUs are active, we have $c=j$. It is also clear that, as MUs arrive and leave, the system state $j$ will change. Due to the CAC of (2), there will be a maximum system state, denoted as $j_{\max }$.

Our goal is to describe the CDMA system as a CTMC and to derive an efficient formula for the calculation of system state probabilities. Based on them, we will then be able to determine the new-call and handoff-call blocking probabilities. To understand the CTMC modeling approach, let us consider a CDMA system that supports $K=2$ service-classes. Assume that $j_{\max }=4$ and, therefore, the system has the following five possible states $j=0,1,2,3$, and 4 . The call load factors of the first service-class are $L_{1, N}=0.003, L_{1, H P}=0.002$, and $L_{1, H P}=0.001$, for new calls, peak-rate handoff calls, and low-rate handoff calls, respectively. Similarly, the corresponding call load factors of the second service-class are $L_{2, N}=0.002, L_{2, H P}=0.002$ and $L_{2, H L}=0.001$. Recall that call load factors can be calculated from (1). Also assume that the rate thresholds are $n_{1, H}=0.003$ and $n_{2, H}=0.002$ for the first and the second service-class, respectively. By selecting $g=0.001$, we get the following resource requirements: $b_{1, N}=$ $3, b_{1, H P}=2, b_{1, H L}=1, b_{2, N}=b_{2, H P}=2, b_{2, H L}=1$. Also, we get the following rate thresholds: $J_{1, H}=3, J_{2, H}=2$.

The state transition diagrams of the CTMC for the first and the second service-classes are shown in Figures 1 and 2, respectively. Some transitions are due to a single type of calls; green arrows for new calls and blue arrows for handoff calls. Some other transitions, depicted by dashed red lines, are due to both types.

In Figures 1 and 2 we use the following notation:

- $\quad \lambda_{k, N}(j)$ : effective transition rate of service-class $k$ new calls from state $(j)$ to state $\left(j+b_{k, N}\right)$

- $\quad \lambda_{k, H}(j)$ : effective transition rate of of service-class $k$ handoff calls from state $(j)$ to state $\left(j+b_{k, H P}\right)$ if $j<J_{k, H}$, or to state $\left(j+b_{k, H L}\right)$ if $j \geq J_{k, H}$

- $\quad \mu_{k, t}$ : service rate of service-class $k$, type $t(t \in$ $\{N, H\})$ calls

- $\quad Y_{k, t r}(j)$ : mean number of service-class $k$, type $t$, rate $r$ calls in state $j$

For example, in Figure 1 we observe that the transitions caused by new calls are always in steps of $b_{1, N}=3$. On the other hand, the transitions caused by handoff calls are more complicated, due to existence of the rate threshold $J_{1, H}=3$. Transitions from states $j<J_{1, H}$ to higher states are in steps of $b_{1, H P}=2$; transitions from states $j \geq J_{1, H}$ to higher states are in steps of $b_{1, H L}=1$. Furthermore, we observe that states $j>j_{\max }-b_{1, N}=5-3=2$ are blocking states for new calls, due to the fact that in these states the available RUs are less than required. As a consequence, transitions from states $j>2$ to higher states are not possible for new calls. Similarly, there is one blocking state, $j=4$, for handoff calls.

The effective transition rate, $\lambda_{k, t}(j)$, depends on the number of idle sources, $S_{k, t}-Y_{k, t r}(j)$, the call arrival rate per idle source, $\lambda_{k, t}$, and the blocking probability in state $j$, denoted as state blocking factor, $F_{k, t}(j)$ :

$$
\lambda_{k, t}(j)=\left(S_{k, t}-Y_{k, t r}(j)\right) \lambda_{k, t}\left(1-F_{k, t}(j)\right)
$$

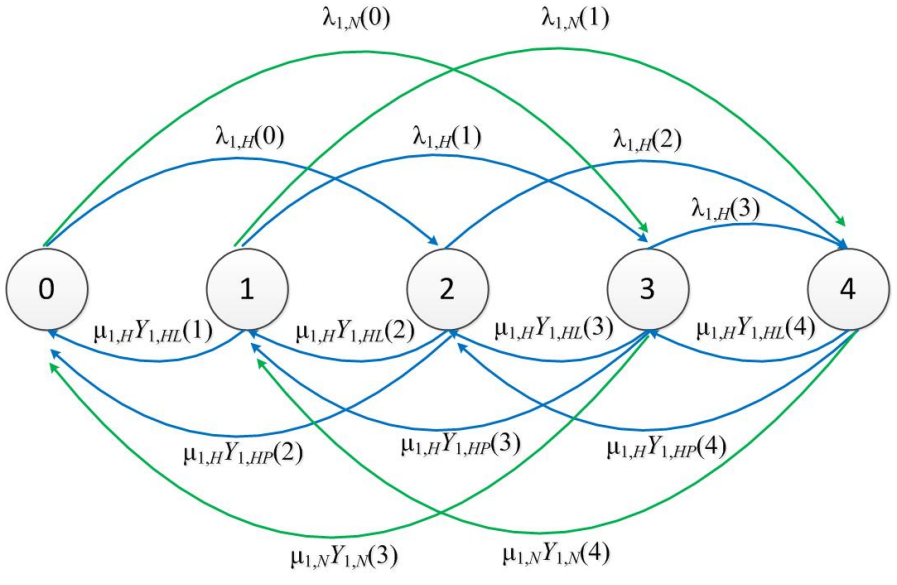

Fig. 1. CTMC for a simple example. First service-class.

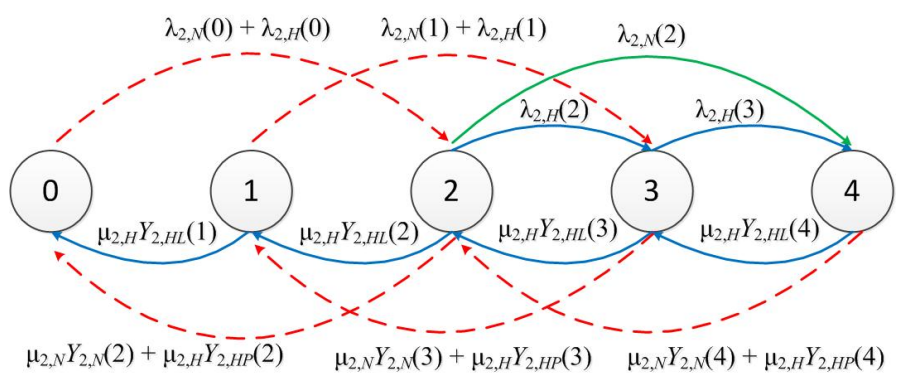

Fig. 2. CTMC for a simple example. Second service-class.

In the following subsection, we calculate the factors $F_{k, t}(j)$ and, consequently, the state probabilities, $q(j), j=0, \ldots, j_{\max }$.

Returning back to the example of Figures 1 and 2, we observe that some transitions to higher states (e.g. $\lambda_{1, H}(0)$ ) are accompanied by corresponding transitions to lower states $\left(\mu_{1, H} Y_{1, H P}(2)\right)$. For cases like that, we can assume the following local balance between to corresponding states:

$$
\lambda_{k, t}(j) q(j)=\mu_{k, t} Y_{k, t r}\left(j+b_{k, t r}\right) q\left(j+b_{k, t r}\right)
$$

for $j=0, \ldots, j_{\max }-b_{k, t r}$

In the figures, we also observe that some transitions to lower states due to handoff calls (e.g. $\mu_{1, H} Y_{1, H L}(1)$ ) have no corresponding transitions to higher states. For cases like that, we will assume that these rates are negligible, because $Y_{1, H L}(1) \rightarrow 0$. In general, we assume the following:

$$
\begin{aligned}
& Y_{k, H L}(j)=0 \text { if } j<J_{k, H} \\
& Y_{k, H P}(j)=0 \text { if } j \geq J_{k, H}
\end{aligned}
$$

Assumptions (7) and (8) introduce some approximation errors. In Section IV, we evaluate the impact of these approximations on the accuracy of the derived results.

\section{Local Blocking Factors}

In this subsection, we derive formulas for the calculation of factors $F_{k, t r}(j)$. First, we define the resource share, $P_{k, t r}(j)$, of service-class $k$, type $t$, rate $r$ calls in a state $j$ as follows:

$$
P_{k, t r}(j)=\frac{Y_{k, t r}(j) b_{k, t r}}{j}
$$


with $t \in\{N, H\}$ and $r \in\{P, L\}$. This equation essentially shows the ratio of RUs occupied by a particular call category over the total number of occupied RUs in a given state $j$.

Next, we define the resource occupancy probability, $\Lambda(c \mid j)$, as the conditional probability that $c$ RUs are occupied in state $j$. The calculation of $\Lambda(c \mid j)$ is given as follows:

$$
\begin{aligned}
& \Lambda(c \mid j)=\sum_{k=1}^{K} \sum_{t} \sum_{r} P_{k, t r}(j)\left[v_{k} \Lambda\left(c-b_{k, t r} \mid j-b_{k, t r}\right)\right. \\
& \left.+\left(1-v_{k}\right) \Lambda\left(c \mid j-b_{k, t r}\right)\right]
\end{aligned}
$$

for $j=1, \ldots, j_{\max }$ and $c \leq j$, with $\Lambda(0 \mid 0)=1$ and $\Lambda(c \mid j)=0$ for $c>j$.

Given the above, we now can determine the state blocking factors as follows:

$$
F_{k, t r}(j)=\sum_{c=0}^{j} \beta_{k, t r}(c) \Lambda(c \mid j)
$$

where $\beta_{k, t r}(c)=\beta_{k, t r}\left(n_{\text {intra }}\right)$ for $c=\left\lfloor\frac{n_{\text {intra }}}{g}\right\rfloor$.

\section{State Probabilities and Call Blocking Probabilities}

Having determined the factors $F_{k, t r}(j)$ in the previous subsection, we can now solve the local balance equations of (7) and derive the state probabilities:

$$
\begin{aligned}
& q(j)=\frac{1}{j} \sum_{k=1}^{K} \sum_{t} \sum_{r}\left[\left(S_{k, t}-Y_{k, t r}(j)+1\right) a_{k, t}(1-\right. \\
& \left.\left.F_{k, t r}\left(j-b_{k, t r}\right) b_{k, t r} \delta_{k, t r}(j) q\left(j-b_{k, t r}\right)\right)\right]
\end{aligned}
$$

for $j=1, \ldots, j_{\max }$ and $q(j)=0$ for $j<0$, where $\sum_{j=0}^{j_{\max }}=1$.

The parameters $\delta_{k, t r}(j)$ are used in (12) to impose the approximations of (8) and are defined as follows:

$$
\begin{aligned}
& \delta_{k, N}(j)=1 \\
& \delta_{k, H P}(j)= \begin{cases}1, & \text { if } j<J_{k, H} \\
0, & \text { otherwise }\end{cases} \\
& \delta_{k, H L}(j)= \begin{cases}1, & \text { if } j \geq J_{k, H} \\
0, & \text { otherwise }\end{cases}
\end{aligned}
$$

The parameters $Y_{k, t r}(j)$, used in (12), can be approximated based on the method of [19]. According to this method, the average number of service-class $k$ calls in state $j$ can be well approximated assuming infinite number of traffic sources for each service-class. Hence we have:

$$
Y_{k, t r}(j) \approx \frac{a_{k, t} q\left(j-b_{k, t r}\right)\left(1-F_{k, t r}\left(j-b_{k, t r}\right)\right)}{q(j)}
$$

Finally, we can calculate the new-call and handoff-call blocking probabilities by adding all the state probabilities multiplied by the corresponding state blocking factors:

$$
\begin{gathered}
B_{k, N}=\sum_{j=0}^{j_{\max }} q(j) F_{k, N}(j) \\
B_{k, H}=\sum_{j=0}^{J_{k, H}-1} q(j) F_{k, H P}(j)+\sum_{j=J_{k, H}}^{j_{\max }} q(j) F_{k, H L}(j)
\end{gathered}
$$

TABLE I. TRAFFIC PARAMETERS

\begin{tabular}{|l|l|l|}
\hline & \multicolumn{1}{|c|}{$1^{\text {st }}$ service-class } & \multicolumn{1}{|c|}{$2^{\text {nd }}$ service-class } \\
\hline & $R_{1, N}=144 \mathrm{Kbps}$ & $R_{2, N}=384 \mathrm{Kbps}$ \\
Bit rate & $R_{1, H P}=144 \mathrm{Kbps}$ & $R_{2, H P}=384 \mathrm{Kbps}$ \\
& $R_{1, H L}=120 \mathrm{Kbps}$ & $R_{2, H L}=320 \mathrm{Kbps}$ \\
\hline Rate threshold & $n_{1, H}=0.4$ & $n_{2, H}=0.4$ \\
\hline Activity factor & $v_{1}=0.67$ & $v_{2}=1$ \\
\hline BER parameter & $\left(E_{b} / N_{0}\right)_{1}=3 \mathrm{~dB}$ & $\left(E_{b} / N_{0}\right)_{2}=4 \mathrm{~dB}$ \\
\hline New sources & $N_{1, N}=100$ & $N_{2, N}=50$ \\
\hline Handoff sources & $N_{1, H}=20$ & $N_{2, H}=10$ \\
\hline
\end{tabular}

TABLE II. OFFERED TRAFFIC LOAD (ERL)

\begin{tabular}{|c|c|c|c|c|}
\hline & \multicolumn{2}{|c|}{$1^{\text {st }}$ service-class } & \multicolumn{2}{c|}{$2^{\text {nd }}$ service-class } \\
\hline traffic point & $a_{1, N}$ & $a_{1, H}$ & $a_{2, N}$ & $a_{2, H}$ \\
\hline 1 & 0.10 & 0.02 & 0.05 & 0.01 \\
\hline 2 & 0.20 & 0.04 & 0.10 & 0.02 \\
\hline 3 & 0.30 & 0.06 & 0.15 & 0.03 \\
\hline 4 & 0.40 & 0.08 & 0.20 & 0.04 \\
\hline 5 & 0.50 & 0.10 & 0.25 & 0.05 \\
\hline 6 & 0.60 & 0.12 & 0.30 & 0.06 \\
\hline 7 & 0.70 & 0.14 & 0.35 & 0.07 \\
\hline 8 & 0.80 & 0.16 & 0.40 & 0.08 \\
\hline
\end{tabular}

\section{PERFormance EVAluation}

In this section, we evaluate the accuracy of our proposed model. We have developed an event-driven simulator using the SIMSCRIPT III simulation tool [20]. We simulated call arrival/departure events to/from the CDMA system according to the description of Section II. After each event, the BS estimates the cell load and possibly blocks an arriving call according to the CAC condition of (2). Next, we implemented the analytical model of Section III. The calculation of blocking probabilities is based on (15) and (16). These calculations are very fast, due to recursive and efficient determination of state probabilities in (12).

We consider $K=2$ independent service-classes with the parameters shown in Table I. Note that, in both service-classes, new and peak-rate handoff calls request the same data rate. In the case of the first service-class, handoff calls request $120 \mathrm{Kbps}$ if the cell load is greater than or equal to the rate threshold $\left(n \geq n_{1, H}\right)$. Similarly handoff calls of the second service-class request $320 \mathrm{Kbps}$ if $n \geq n_{2, H}$. We use the following CAC thresholds: $n_{\max , N}=0.8$ and $n_{\max , H}=0.7$, for new and handoff calls, respectively. The offered traffic load per idle source of the two service-classes is shown in Table II.

We compare the analytical versus simulation blocking probabilities for both new and handoff calls. These blocking probabilities versus the offered traffic-load are shown in Figs. 3 and 4. Simulation results are mean values of 10 runs with $95 \%$ confidence interval. The resultant reliability ranges of the simulation measurements are very small and, therefore, we present only mean values. We observe that the accuracy of the proposed analytical model is satisfactory, as the analytical results are very close to simulation results. We also observe that, as expected, the blocking probabilities of handoff calls are lower than the blocking probabilities of new calls. This is due to the fact that we used a higher CAC threshold for the handoff calls in comparison with the CAC thresholds for new calls. In our experiments, we have also considered other scenarios, with a larger number of service-classes, different data rates, and rate thresholds. Due to space limitations, these results are not presented. However, in all cases the accuracy 


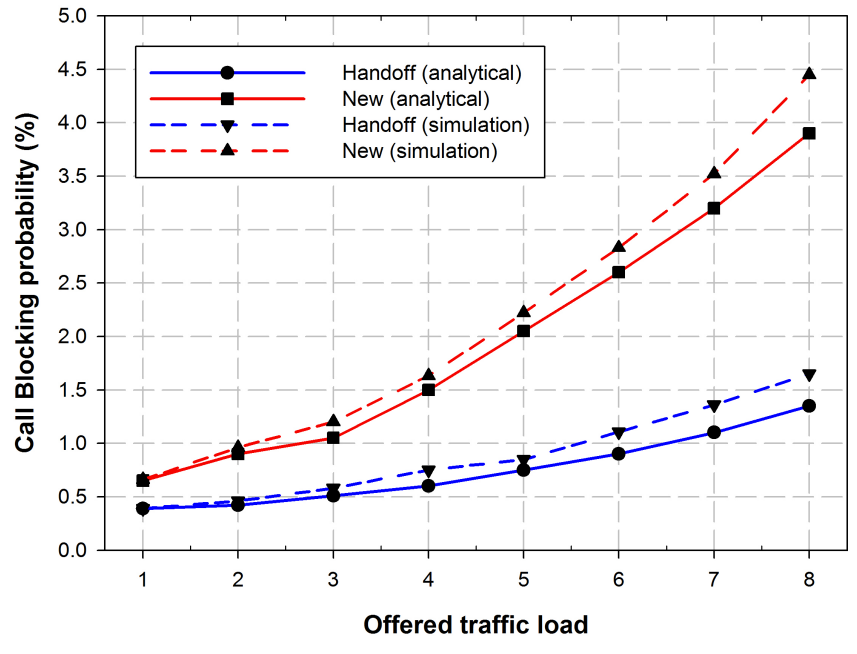

Fig. 3. Call blocking probabilities vs offered traffic-load (1st service-class).

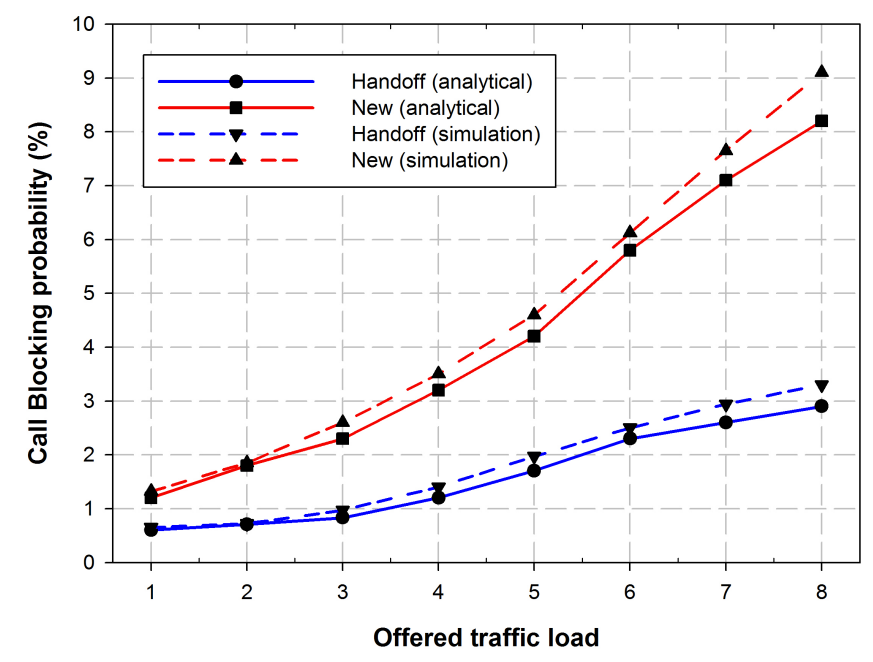

Fig. 4. Call blocking probabilities vs offered traffic-load (2nd service-class).

of the proposed model is as in Figs. 3 and 4.

\section{CONCLUSion}

In this paper, we have present a novel modelling approach for handoff traffic in cellular CDMA systems. When the system is overloaded, handoff calls may request for less bandwidth, according to a predefined threshold. The applied admission policy ensures that the handoff-call blocking probability can be significantly reduced. We also consider a finite number of traffic sources for each service-class. Our model is described by a continuous-time Markov chain and results in an approximate but recurrent and efficient formula for system state probabilities. Our simulation experiments show that the accuracy of the proposed model is satisfactory. In our future work, we plan to investigate the applicability of the proposed approach in the downlink of CDMA and in systems with hard capacity.

\section{REFERENCES}

[1] S. Chen, J. Zhao, "The requirements, challenges, and technologies for $5 \mathrm{G}$ of terrestrial mobile telecommunication," IEEE Commun. Mag., vol. 52, no. 5, pp.36-43, 2014.

[2] Z. Wang, S. Fan, Y. Rui, "CDMA-FMT: A novel multiple access scheme for 5G wireless communications," Proc. 19th International Conference on Digital Signal Processing, Hong Kong, Aug. 2014.

[3] D. Staehle, A. Mäder, "An analytic approximation of the uplink capacity in a UMTS network with heterogeneous traffic," Proc. ITC-18, Berlin, Germany, Sept. 2003.

[4] G. A. Kallos, V. G. Vassilakis, I. D. Moscholios, M. D. Logothetis, "Performance modelling of W-CDMA networks supporting elastic and adaptive traffic," Proc. 4th HET-NETs 2006, Ilkley, U.K., Sept. 2006.

[5] V. G. Vassilakis, G. A. Kallos, I. D. Moscholios, M. D. Logothetis, "The wireless Engset multi-rate loss model for the call-level analysis of W-CDMA networks," Proc. 18th IEEE PIMRC, Athens, Greece, Sept. 2007.

[6] M. Stasiak, A. Wisniewski, P. Zwierzykowski, M. Glabowski, "Blocking probability calculation for cellular systems with WCDMA radio interface servicing PCT1 and PCT2 multirate traffic," IEICE Trans. on Commun., vol. E92-B, no. 4, 2009, pp. 1156-1165.

[7] V. G. Vassilakis, G. A. Kallos, I. D. Moscholios, M. D. Logothetis, "Evaluation of a call admission control scheme in W-CDMA cellular networks," Proc. 6th IEEE CSNDSP, Graz, Austria, July 2008.

[8] V. G. Vassilakis, G. A. Kallos, I. D. Moscholios, M. D. Logothetis, "Call-level analysis of W-CDMA networks supporting elastic services of finite population," Proc. IEEE ICC-2008, Beijing, China, May 2008.

[9] G. A. Kallos, V. G. Vassilakis, M. D. Logothetis, "Call-level performance analysis of a W-CDMA cell with finite population and interference cancellation," European Trans. on Telecomm., vol. 22, no. 1, 2011, pp. 25-30.

[10] I. D. Moscholios, M. A. Katsiva, G. A. Kallos, V. G. Vassilakis, M. D. Logothetis, "Equalization of congestion probabilities in a W-CDMA cell supporting calls of finite sources with interference cancellation," Proc. IEEE/IET 9th CSNDSP, Manchester, U.K., July 2014.

[11] I. B. Daskalopoulos, V. G. Vassilakis, M. D. Logothetis, "Thorough analysis of downlink capacity in a WCDMA cell," Proc. 1st International Conference on Mobile Lightweight Wireless Systems (MOBILIGHT), Athens, Greece, May, 2009.

[12] M. Stasiak, P. Zwierzykowski, D. Parniewicz, "Modelling of the WCDMA interface in the UMTS network with soft handoff mechanism," Proc. IEEE GLOBECOM-2009, Honolulu, USA, Dec. 2009.

[13] S. Hanczewski, M. Stasiak, P. Zwierzykowski, "A new model of the soft handover mechanism in the UMTS network," Proc. IEEE/IET 9th CSNDSP, Manchester, U.K., July 2014.

[14] V. G. Vassilakis, M. D. Logothetis, "The wireless Engset multirate loss model for the handoff traffic analysis in W-CDMA networks," Proc. 19th IEEE PIMRC, Cannes, France, Sept. 2008.

[15] V. G. Vassilakis, G. A. Kallos, I. D. Moscholios, M. D. Logothetis, "On the handoff-call blocking probability calculation in W-CDMA cellular networks," Proc. 4th IARIA AICT, Athens, Greece, June 2008.

[16] I. D. Moscholios, G. A. Kallos, V. G. Vassilakis, M. D. Logothetis, "Congestion probabilities in CDMA-based networks supporting batched Poisson input traffic," Wireless Personal Communications, vol. 79, no. 2, pp. 1163-1186, Nov. 2014.

[17] D. Xenakis, N. Passas, L. Merakos, C. Verikoukis, "Mobility management for femtocells in LTE-Advanced: key aspects and survey of handover decision algorithms," IEEE Comm. Surv. \& Tut., vol. 16, no. 1, pp. 64-91, 2014.

[18] J. Kaufman, "Blocking with retrials in a completely shared resource environment," Performance Evaluation, vol. 15(2), 1992, pp. 99-113.

[19] M. Glabowski, M. Stasiak, "An approximate model of the fullavailability group with multi-rate traffic and finite source population," Proc. MMB\&PGTS'04, Dresden, Germany, Sept. 2004, pp. 195-204.

[20] Simscript III, http://www.simscript.com [October 2014]. 